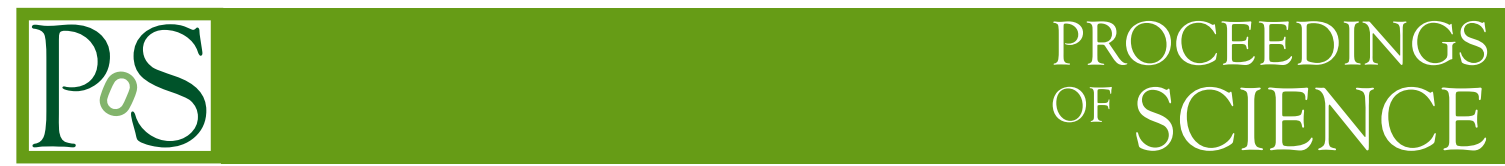

\title{
W mass and width from LEP
}

\author{
Ambreesh Gupta*t \\ University of Chicago, USA \\ E-mail: agupta@hep.uchicago.edu
}

We present a measurement of the mass and width of the $\mathrm{W}$ boson by direct reconstruction in the $q \bar{q} q \bar{q}$ and $q \bar{q} \ell^{ \pm} v_{\ell}$ channels by of the four LEP 2 experiments, ALEPH, DeLPHI, L3 and OPAL. Each experiment used about $700 \mathrm{pb}^{-1}$ of collected data. Major systematic uncertainties are discussed.

International Europhysics Conference on High Energy Physics

July 21st - 27th 2005

Lisboa, Portugal

*Speaker.
$\dagger$ For the LEP Collaborations 


\section{Introduction}

Measurement of the $\mathrm{W}$ mass was one of the principal goals of the LEP 2 program at CERN. We report here a new combined preliminary measurement of W mass and width from the four LEP experiments ALEPH, L3, DeLPHI and OPAL [1]. The combination uses the final W mass and width measurement from OPALand preliminary results from the other three experiments.

\section{Event Reconstruction and W Mass and Width Measurement}

At LEP 2, W bosons are produced in pairs. During the years 1996-2000, LEP ran at centerof-mass energies, above the WW production threshold, in the range 161-209 GeV. The experiments collected about $700 \mathrm{pb}^{-1}$ each. In the Standard Model, WW events decay into fully leptonic $\ell v \ell v(10.6 \%)$, semi leptonic qq $\ell v(43.9 \%)$, and fully hadronic qqqq(45.6\%) final states. At WW production threshold energy $\sqrt{s}=161 \mathrm{GeV}$, WW production cross-section is sensitive to $\mathrm{W}$ mass, which was used to measure the $\mathrm{W}$ mass $=80.40 \pm 0.22 \mathrm{GeV}$ [2].

Most of the LEP 2 data is at higher energies and $\mathrm{W}$ mass and width are measured by direct reconstruction of the $\mathrm{W}$ boson invariant mass distribution. The invariant mass of the two $\mathrm{W}$ bosons are kinematically reconstructed from the measured energy and direction of jets and leptons in the event. Since, the LEP beam energy is very precisely measured it is used as kinematic constraint to the event. This improves the reconstructed invariant mass resolution by a factor of 2-3.

The fully hadronic events are characterized by 4 hadronic jets in the event with little or no missing energy. Since there are no unmeasured quantities in these events, four constraints from energy and momentum conservation can be used. Also, since the resolution of reconstructed mass is much larger than the $\mathrm{W}$ width, the two reconstructed masses can be constrained to be equal. The qqqq events have the highest branching fraction, but the sensitivity of this channel is reduced due to the following three factors - Significant background from two fermion $Z^{0} \rightarrow q \bar{q}$, combinatorial background due to ambiguity in assigning the correct pair of jets to the two W's and large systematics due to final state interactions.

The semi-leptonic events, characterized by two distinct hadronic jets, a high-momentum lepton (electron, muon or tau) and missing momentum due to the neutrino, form the most important channel for $\mathrm{W}$ mass measurement. The channel has high efficiency and low backgrounds. In these semi-leptonic events, since the neutrino is unmeasured, the effective number of constraints is reduced to one, or two if the equal mass constraint is used. The fully leptonic channel with two unobserved neutrinos has limited mass information. OPAL and ALEPH have measured $\mathrm{W}$ mass in this channel [2].

There are three techniques to extract $M_{\mathrm{W}}$ and $\Gamma_{\mathrm{W}}$ from the reconstructed invariant mass spectrum. The most widely used (ALEPH, L3, OPAL) involves re-weighting fully simulated MonteCarlo events to an arbitrary value of $M_{\mathrm{W}}$ and $\Gamma_{\mathrm{W}}$ using the ratio of 4 fermion matrix elements. The second method (DELPHI, OPAL) constructs an event likelihood by convolving a Breit-Wigner with Initial State Radiation (ISR) and detector resolution function. Any mis-modeling is corrected using fully simulated Monte-Carlo events. The third method (OPAL) fits an asymmetric Breit-Wigner to the reconstructed mass spectrum. The width of the Breit-Wigner is adjusted to model the ISR and detector effects. Again, any mis-modeling is corrected using fully simulated events. 


\section{Systematic Uncertainties}

The most important uncertainties on are related to the the LEP beam energy, detector response, hadronization and final state interaction (FSI) modeling. The four LEP collaborations combine their results taking account of systematic uncertainties which are correlated between channels, experiments and years of LEP running. A summary of the uncertainties is given in table 1 .

\begin{tabular}{l|r|r|r|r}
\hline & \multicolumn{3}{|c|}{$\Delta M_{W}(\mathrm{MeV})$} & $\Delta \Gamma_{W}(\mathrm{MeV})$ \\
\hline Systematics source & $\mathrm{qq} \ell v$ & $\mathrm{qqqq}$ & $\mathrm{qq} \ell v+\mathrm{qqqq}$ & $\mathrm{qqqq}+\mathrm{qq} \ell v$ \\
\hline Hadronization & 17 & 18 & 17 & 34 \\
Color Reconnection & - & 49 & 7 & 14 \\
Bose-Einstein Cor. & - & 22 & 3 & 25 \\
Detector & 14 & 8 & 13 & 31 \\
ISR/FSR & 10 & 9 & 10 & 14 \\
LEP Beam Energy & 14 & 11 & 13 & 5 \\
Others & 4 & 5 & 4 & 19 \\
\hline Total Systematics & 28 & 62 & 28 & 59 \\
\hline Statistical & 31 & 48 & 27 & 65 \\
\hline Total Uncertainty & 42 & 79 & 39 & 88 \\
\hline
\end{tabular}

Table 1: Systematics and statistical uncertainties for $\mathrm{W}$ mass and width measurement at LEP.

LEP Beam Energy: At LEP 2 the beam energy is measured by measuring the bending magnetic field with the help of precise NMR probes in the LEP ring. It is calibrated with resonant depolarization technique by extrapolating to lower energies $(40-60 \mathrm{GeV})$. The final result is described in [3].

Detector Effects: To calibrate the detector response to jets and leptons, the experiments use large samples of $Z^{0}$ resonance data that are taken at different times of each year of LEP 2 running. For both jets and leptons, a final calibration of scale and resolution is derived by comparing $Z^{0}$ data and Monte-Carlo. Systematics due to modeling of jet mass are also estimated.

Hadronization: Hadronization uncertainties are due to mis-modeling of final state quark $\mathrm{W} \rightarrow \mathrm{q} \overline{\mathrm{q}}$ decay in to jets of hadrons. The experiments use different hadronization models ( PYTHIA, ARIADNEand HERWIG) to estimate these uncertainties. All models have been tuned separately by each experiment using the $\mathrm{Z}^{0}$ data. Differences in the models due to rate and reconstruction of kaons and baryons and taken care by re-weighting.

Final State Interaction: The decay products of two W's could interact via Color Reconnection (CR) and Bose-Einstein Correlations (BEC) and produce a shift in W mass and width measurement. Several theoretical models of CR in W-pair events exist, of which the most commonly used are the SK1, ARIADNE (AR2) and HERWIG models [2]. The SK1 model has a has an adjustable free parameter to model amount of CR, which gives $\mathrm{W}$ mass shift of $300 \mathrm{MeV}$ for full reconnection. Although data disfavors full reconnection in SK1 by more than five standard deviations, it does not rule out no color reconnection scenario. The upper end of $68 \%$ confidence interval on the SK1 parameter is used to set estimate uncertainty on $\mathrm{W}$ mass and width. Also, to reduce sensitivity color reconnection, the jet direction is recalculated excluding soft tracks. The final OPAL analysis 

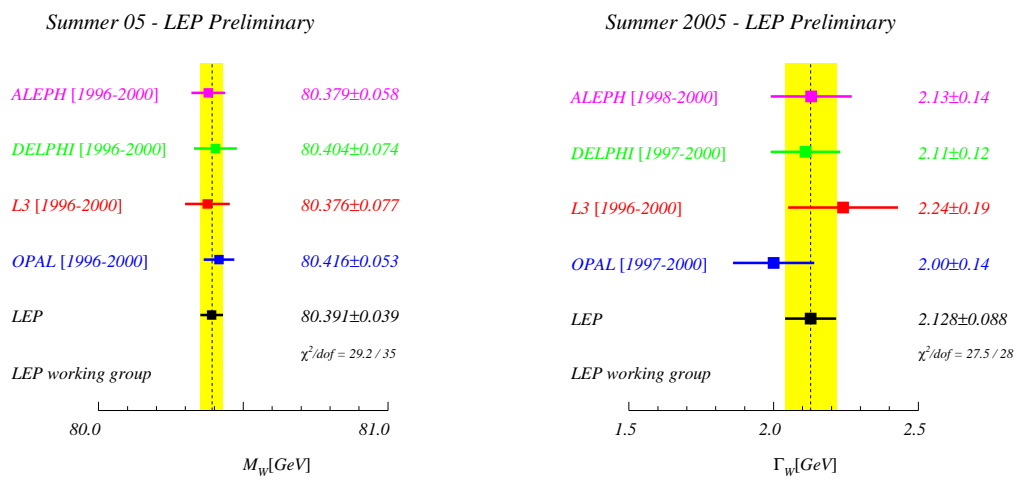

Figure 1: Preliminary results on the $W$ mass and width from LEP.

applied a cut of $2.5 \mathrm{GeV}$, which reduced the $\mathrm{W}$ mass shift from $100 \mathrm{MeV}$ to $41 \mathrm{MeV}$. The loss in statistical sensitivity was about $18 \%$. Similar techniques by other experiments in their final analysis will further reduce the overall CR uncertainty.

The Bose-Einstein correlations lead to an enhancement in the production of pairs of identical bosons close in phase space. The effect of this correlation has been clearly seen between pairs of particles within the same (intra) $\mathrm{Z}$ or $\mathrm{W}$ boson. The inter $\mathrm{W}$ correlation is shown to be only a fraction of the intra-W correlation [2]. Similar to CR, removing soft tracks reduces the W mass systematics due to BEC.

\section{Summary and Results}

Based on the analysis of the entire LEP 2 data sample, a new preliminary $\mathrm{W}$ mass and the width from LEP is

$$
\begin{gathered}
M_{\mathrm{W}}=80.391 \pm 0.027 \text { (stat.) } \pm 0.028 \text { (syst.) GeV } \\
\Gamma_{\mathrm{W}}=2.128 \pm 0.065 \text { (stat.) } \pm 0.059 \text { (syst.) GeV }
\end{gathered}
$$

The result from each experiment are shown in fig 1. The final OPAL result combines $161 \mathrm{GeV}$ threshold measurement nd $\ell v \ell v$ channel measurement [2]. Compared to previous combination, the LEP uncertainty is lowered to $39 \mathrm{MeV}$. ALEPH, DELPHI and L3 results do not include the final LEP beam energy and the final FSI analysis, which will further reduce the LEP W mass uncertainty.

\section{References}

[1] AlePH collaboration, Nucl. Inst. Meth. A 294, 121 (1990); L3 Collaboration, Nucl. Inst. Meth. A 289, 35 (1990); DELPHI Collaboration Nucl. Inst. Meth. A 303, 233 (1991); OpAL Collaboration Nucl. Inst. Meth. A 305, 275 (1991).

[2] ALEPH, DELPHI, L3, and OPAL collaborations, LEP electroweak group and SLD heavy flavor group, hep-ex/0511027.

[3] LEP Energy Working Group, Eur. Phys. J. C39 253 (2005). 